\title{
Anxious Personality and Breast Cancer: Possible Negative Impact on Quality of Life After Breast-Conserving Therapy
}

\author{
Alida F. W. van der Steeg • Jolanda De Vries • \\ Jan A. Roukema
}

Published online: 20 March 2010

(c) The Author(s) 2010. This article is published with open access at Springerlink.com

\begin{abstract}
Background Quality of Life (QoL) is an important outcome measure in oncology. To assess the influence of surgical treatment and personality on QoL in women with breast cancer, a longitudinal prospective cohort study was done.

Methods Women $(n=222)$ completed questionnaires concerning QoL (WHOQOL 100) and personality (NEOFFI and STAI) prior to the diagnosis of breast cancer and 1,3, 6 , and 12 months after diagnosis and treatment. One hundred five women were treated with breast-conserving therapy (BCT) and 117 women underwent mastectomy (MTC). Results The two treatment groups did not differ on overall QoL. At all measurement times the influence of trait anxiety on overall QoL was substantial in the BCT group. Women with a high score on trait anxiety were seven times more likely to have a low overall QoL 1 year after BCT. In the MTC group overall QoL was influenced mainly by neuroticism. Conclusions Personality, especially trait anxiety and neuroticism, determined patients' overall QoL scores.
\end{abstract}

A. F. W. van der Steeg · J. A. Roukema

Department of Surgery, St. Elisabeth Hospital, P.O. Box 90151,

5000 LC Tilburg, The Netherlands

J. De Vries · J. A. Roukema

Department of Psychology and Health, Medical Psychology,

Tilburg University, P.O. Box 90153, 5000 LE Tilburg,

The Netherlands

A. F. W. van der Steeg $(\bowtie)$

Pediatric Surgical Center Amsterdam, Emma Children's

Hospital, AMC and VU University Medical Center,

P.O. Box 22660, 1100 DD Amsterdam, The Netherlands

e-mail: A.F.vanderSteeg@amc.uva.nl

A. F. W. van der Steeg - J. De Vries - J. A. Roukema CoRPS, Center of Research on Psychology in Somatic diseases,

Tilburg University, Tilburg, The Netherlands
Women with an anxious personality fared worse concerning QoL after breast conserving therapy.

\section{Introduction}

Breast cancer is the most frequently diagnosed cancer in women in the developed countries. In the Netherlands, $11 \%$ of women will be diagnosed with breast cancer [1]. Primary treatment for breast cancer is surgery. In earlystage breast cancer, i.e., a tumor smaller than $5 \mathrm{~cm}$ in diameter and no metastases beyond the axilla (stages I and II), a woman can choose between breast-conserving therapy (BCT) and mastectomy (MTC). BCT refers to lumpectomy and a staging operation of the axillary lymph nodes followed by radiation therapy of the remaining breast. In MTC, all breast tissue, including the nipple, is removed and a staging operation is performed on the axillary lymph nodes. Depending on tumor characteristics and the presence of axillary metastases, surgery can be followed by adjuvant chemotherapy, hormonal therapy, and/or radiation of the area around the collar bone. The two surgical treatment modalities have proven to be equal with respect to long-term survival, although disease-free survival is less after conserving surgery [2].

Since 1929 physicians have been studying the possibilities and results of breast preservation [3]. In many instances the predominant reason for its use was that patients refused the recommended radical mastectomy [4]. Since the long-term follow-up studies from randomized trials have been published [2], the general opinion among professional caregivers is that $\mathrm{BCT}$ is the more favorable treatment option since it is less mutilating and, therefore, may lead to a better quality of life (QoL) compared to the QoL after a MTC. 
QoL is an important outcome measurement when evaluating the results of cancer treatment. It is a multidimensional concept that measures the satisfaction of a person with a wide range of aspects [5]. QoL has been studied extensively in breast cancer patients, and the influence of surgical treatment on QoL has been addressed. It is known that QoL is influenced by health [5], culture, socioeconomic status, and personality [6]. So far, the relationship between personality and breast cancer has been studied only once [7]. This study refers to the same study population as the present study and showed that trait anxiety had a profound influence on overall QoL and was even more important for QoL than the diagnosis of breast cancer. The relationship between surgical treatment, personality, and overall QoL in breast cancer patients has not been studied before.

In the present study, QoL was assessed in a prospective longitudinal setting. Two hypotheses based on previous publications were studied [7, 8]: (1) compared with MTC, BCT results in a better QoL and (2) personality, and especially trait anxiety, will have a significant influence on QoL for both treatment groups. The major differences between this study and previously published studies are that there was a baseline measurement before diagnosis, focusing on the role of personality factors, and a questionnaire that assesses all aspects of QoL was used.

\section{Patients and methods}

\section{Patients}

Women who were referred to the specialized mamma-care nurse of the St. Elisabeth Hospital, the Maasland Hospital (patient accrual since August 2004), or the Jeroen Bosch Hospital (patient accrual since January 2006) with a palpable lump in the breast or an abnormality on screening mammography between September 2002 and January 2007 were asked to participate in the study. Women who had a medical history of either benign breast disease or breast cancer were excluded from the study.

Eight hundred ninety-four women were eligible for participation in this study. Six hundred eight women $(68 \%)$ gave informed consent and completed the first set of questionnaires. Reasons for refusal were the length of the questionnaires and the amount of stress the women experienced, which they felt impaired their concentration while completing the questionnaires. There were no differences in clinical characteristics (e.g., age, tumor size) between participants and nonparticipants.

After written informed consent was given and before the first appointment with the surgeon, i.e., before the diagnosis was known, the women completed the first set of questionnaires. Subsequently, questionnaires were also completed 1, 3, 6, and 12 months after diagnosis and treatment. Participation of the patients in the study was not known by the treating surgeon and, therefore, the surgeon did not have any influence on the treatment and clinical follow-up.

\section{Procedures}

The first physical examination was performed by the specialized mamma-care nurse. When a woman presented with locally advanced breast cancer or a palpable tumor that would be too large for BCT if breast cancer is diagnosed, she was asked not to participate in the study. All other women were asked to participate by the mamma-care nurse. The treating surgeon was not involved in the study and, as a standard policy, did not discuss the study with the women. Thus, the surgeon could stay professional in supporting the patient in the decision process.

Between the consult with the nurse and the subsequent mammogram and possible ultrasound, women usually had to wait 90 min during which time the baseline questionnaires were completed. After diagnosis, women who refused operation, women who had a tumor too large for BCT, or women who were treated with neoadjuvant chemotherapy or hormone therapy were removed from the study population. Three women refused surgery, and, based on the initial clinical aspects of the tumor (i.e., $<5 \mathrm{~cm}$ in diameter and no metastasis beyond the ipsilateral axilla), all women were eligible for BCT. This study started in 2002. Oncoplastic surgery was still being developed so it was not implemented in the everyday practice of the participating hospitals. In later years these procedures have been used but not during the inclusion period of this study.

Before deciding which treatment to take, three important aspects were discussed with the patient and her partner: (1) Overall survival is equal for BCT and MTC. (2) Diseasefree survival after BCT may be shorter, with a recurrence rate of about $15 \%$ in 20 years of follow-up [2]. This recurrence rate is irrespective of tumor size, but does relate to the accuracy of the operation. (3) Radiotherapy is mandatory after BCT and may not be necessary after MTC depending on the presence of metastases in the axillary region. When it was clear that the patient understood these aspects, she was allowed to choose her surgical treatment.

\section{Questionnaires}

QoL was assessed using the World Health Organization Quality of Life assessment instrument-100 (WHOQOL-100) [9, 10], a cross culturally developed generic multidimensional questionnaire. It assesses QoL in six domains (Physical health, Psychological health, Level of independence, 
Social relationships, Environment, Spirituality) and a general evaluative facet (Overall quality of life and general health). The reliability and validity of the instrument are good [11].

Personality was assessed at baseline using the Neuroticism-Extraversion-Openness Five Factor Inventory (NEOFFI) $[12,13]$, and the trait anxiety scale of the State-andTrait-Anxiety-Inventory (STAI) [14, 15]. The NEO-FFI was developed to study an individual's personality. Personality is assessed in the five domains of the Five Factor Model: neuroticism, extraversion, openness, agreeableness, and conscientiousness. The psychometric properties are good [16]. The STAI was originally developed to investigate anxiety phenomena in "normal" adults, but it has also proven useful in medical and surgical patients. It measures two types of anxiety. Trait anxiety, used in the present study, concerns differences in individuals in the disposition to respond to stressful situations with varying amounts of stress. The psychometric characteristics of this questionnaire are well established and considered good [17].

Patients were also asked to complete some questions concerning demographic factors such as age, marital status, education, and social economic status. Clinical data were obtained from the medical records of the included patients.

\section{Statistical procedure}

Frequencies were used to present demographic information on the patients before diagnosis. One-way analyses of variance and $\chi^{2}$ tests were used to compare the patients in the BCT, MTC, and conversion (eventually received MTC, although first choice was BCT) groups on baseline characteristics. The predictors of overall QoL were found using the scores on QoL as the dependent variable and the demographic (block one), clinical (block 2), and personality characteristics (block 3 ) as independent variables in a multivariable regression analysis. The variables that significantly influenced overall QoL were then dichotomized (high or not-high score) and subsequently entered into a logistic regression analysis to compute the odds ratio for a low overall QoL score. General linear model for repeated measures (GLM) was used to examine scores on overall QoL over time (1) in patients with BCT and MTC and (2) in patients high or not on trait anxiety in combination with surgical treatment (BCT or MTC + ). In each analysis the characteristics on which the groups appeared to differ at baseline were used as covariates. $\eta^{2}$ is an effect size that can be derived from the output of the GLM. An $\eta^{2}$ between 0.01 and 0.06 is a small effect, between 0.06 and 0.13 is a moderate effect, and 0.14 or higher is a large effect [18]. We needed to include at least 55 patients in one of the groups to reach a power of 0.80 , given that we expected to find a moderate effect size. When differences were found in the GLM between groups, analyses of variance were used to examine QoL differences between groups at one particular measurement time. Again, the characteristics on which the groups differed at baseline were incorporated in the analyses as covariates. A $P<0.05$ is considered statistically significant. All analyses were performed using the SPSS v14.0 (SPSS, Inc., Chicago, IL).

\section{Results}

Of the 608 women who entered the study, 225 were diagnosed with early-stage breast cancer (i.e., tumor $<5 \mathrm{~cm}$ in diameter and no metastases beyond the ipsilateral axilla) and thus could choose between BCT and MTC surgery. Three patients refused operation and were not included in the analyses, leaving 222 patients. The remaining 383 women appeared to have benign breast problems and were left out of the analyses.

One hundred thirty-three women chose BCT and 89 patients opted for MTC. In two of the three participating hospitals, immediate reconstruction after MTC is not offered to the patients. However, when women request immediate reconstruction, it is possible. None of the included patients in any of the three hospitals underwent immediate reconstruction. Due to incomplete removal of the tumor or multifocality established by pathological examination, 57 BCT patients needed additional surgery of whom 28 eventually had complete removal of the breast. Whenever possible, these 57 women had the choice between breast-conserving surgery and mastectomy. These subsequent surgeries were performed within 3-4 weeks after the first operation. This latter group is referred to as the conversion group.

There were no differences between the BCT, the MTC, and the conversion group with regard to demographic factors (Table 1). With respect to clinical factors there were significant differences. Women in the MTC group and the conversion group had larger tumors on radiology and after surgery. A larger percentage of the women in the MTC group were diagnosed with axillary metastases and thus received chemotherapy and/or hormonal therapy more often than the BCT and conversion groups. Since radiotherapy is part of the conserving treatment, the BCT group received radiotherapy more often compared with the other two groups (Table 1). Radiotherapy of the axilla and the area of the ipsilateral clavicula was done when more than three lymph nodes contained metastasis; this was the situation in 5 women in the BCT group, 19 women in the MTC group, and 5 women in the conversion group.

The groups did not differ with regard to overall QoL or the QoL domains. With regard to personality, the conversion group scored significantly lower on neuroticism 
Table 1 Demographics and clinical factors for the three treatment groups

\begin{tabular}{|c|c|c|c|c|}
\hline & $\mathrm{BCT}(n=105)$ & $\operatorname{MTC}(n=89)$ & $\mathrm{BCT}>\mathrm{MTC}(n=28)$ & $P$ value \\
\hline \multicolumn{5}{|l|}{ Demographics } \\
\hline Age & $58.3(8.7 / 0.9)$ & $58.4(9.3 / 1.0)$ & $58.7(10.7 / 2.0)$ & \\
\hline Partner & $88(83.8)$ & $69(77.5)$ & $22(78.6)$ & \\
\hline Children & $87(82.9)$ & $77(86.5)$ & $24(85.7)$ & \\
\hline Educational level $<10 / 10-14 />14$ years & $38(36.9) / 46(44.7) / 19(18.4)$ & $30(35.7) / 40(47.6) / 14(16.7)$ & $14(51.9) / 8(29.6) / 5(18.5)$ & \\
\hline Paid work & $40(38.5)$ & $37(42.5)$ & $9(32.1)$ & \\
\hline \multicolumn{5}{|l|}{ Clinical factors } \\
\hline Tumor size radiology (mm) & $14.9(8.6)$ & $21.0(12.6)$ & $17.3(8.6)$ & 0.001 \\
\hline Tumor size pathology (mm) & $14.1(6.9)$ & $21.7(11.1)$ & $28.7(19.0)$ & $<0.001$ \\
\hline Axillary metastases & $26(25.2)$ & $37(42.0)$ & $10(35.7)$ & \\
\hline Chemotherapy & $19(18.3)$ & $36(40.4)$ & $7(25.2)$ & 0.005 \\
\hline Hormone therapy & $31(29.5)$ & $46(51.7)$ & $8(28.6)$ & 0.002 \\
\hline Radiotherapy & $97(92.4)$ & 17 (19.1) & 5 (17.9) & $<0.001$ \\
\hline
\end{tabular}

Except for age and tumor size, percentages are in parentheses. For age, standard deviation and standard error of the mean are given in parenthesis. For the calculation of the percentage, missings are not included

Table 2 Scores on personality factors and QoL domains at baseline for the three treatment groups

Scores are means. Standard deviation is given in parenthesis

\begin{tabular}{|c|c|c|c|c|}
\hline & BCT & MTC & $\mathrm{BCT}>\mathrm{MTC}$ & $P$ value \\
\hline \multicolumn{5}{|l|}{ Personality } \\
\hline Neuroticism & $30.6(7.5)$ & $30.2(7.5)$ & $26.5(4.4)$ & 0.044 \\
\hline Neuroticism high score & $42.4(3.2)$ & $42.2(2.2)$ & 40.0 & \\
\hline Neuroticism not-high score & $28.3(5.6)$ & $28.2(6.1)$ & $25.9(3.3)$ & \\
\hline Extraversion & $40.6(5.7)$ & $41.9(5.5)$ & $41.5(5.4)$ & \\
\hline Openness to experiences & $36.0(6.6)$ & $34.9(5.1)$ & $35.9(5.3)$ & \\
\hline Agreeableness & $43.7(4.3)$ & $43.3(4.9)$ & $43.6(3.1)$ & \\
\hline Conscientiousness & $45.9(5.6)$ & $45.9(5.3)$ & $45.2(5.0)$ & \\
\hline Trait anxiety & $40.1(11.1)$ & $39.0(11.2)$ & $36.9(9.5)$ & \\
\hline Trait anxiety high score & $52.4(6.6)$ & $51.2(5.3)$ & $48.1(5.1)$ & \\
\hline Trait anxiety not-high score & $33.1(5.8)$ & $32.1(6.9)$ & $31.3(5.2)$ & \\
\hline \multicolumn{5}{|l|}{ QoL domains } \\
\hline Overall QoL and general health & $15.5(2.4)$ & $15.8(2.8)$ & $15.9(2.0)$ & \\
\hline Physical domain & $14.1(2.5)$ & $14.2(2.5)$ & $14.6(2.1)$ & \\
\hline Psychological domain & $14.7(1.8)$ & $14.8(2.1)$ & $15.0(1.4)$ & \\
\hline Social domain & $16.2(2.2)$ & $16.6(2.0)$ & $16.4(2.1)$ & \\
\hline
\end{tabular}

compared with the BCT and MTC groups $(P=0.044)$ (Table 2).

Because there were no differences between the MTC group and the conversion group with respect to demographic factors, clinical factors, QoL, and personality factors, with the exception of neuroticism, these two groups were merged $(\mathrm{MTC}+)$ in further analyses. The intent-totreat principle was not followed since we were interested in the effects of BCT and MTC on QoL. Keeping the conversion group in the BCT group would provide blurred information on this issue.

There were no significant changes over time, nor significant differences between the two treatment groups for overall QoL (Fig. 1) and the three QoL domains (physical, psychological, and social domain).

Within the BCT group in particular, trait anxiety predicted overall QoL (Table 3). Up to $43 \%$ of variance in scores was explained by trait anxiety $(P<0.001)$. Other contributing factors such as agreeableness and education were responsible for less than $10 \%$ of variance. Twelve months after surgery, neuroticism was the main factor of influence in the BCT group and responsible for $34 \%$ of the variance in QoL scores $(P<0.001)$. Demographic factors, the remaining personality factors, and psychological factors did not significantly predict overall QoL in the BCT group. A high score on trait anxiety had a profound negative 


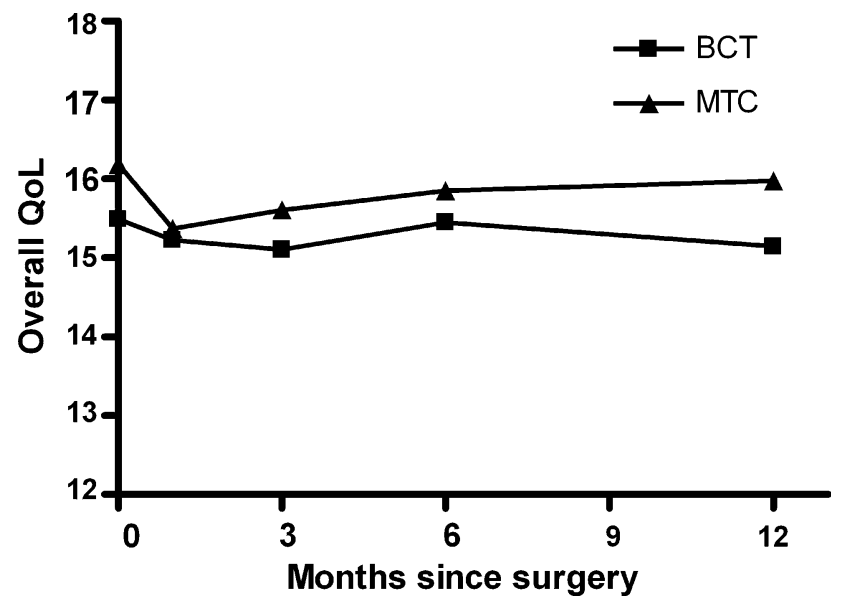

Fig. 1 Scores on overall quality of life and general health. Scores are represented as means for both treatment groups

influence on overall QoL. The subsequent logistic regression analysis showed that the chance that patients in the BCT group with a high score on trait anxiety experienced a low QoL was significantly elevated on all measurement moments, for instance, 7.8 at 6 months postsurgery (odds ratio $[\mathrm{OR}]=7.81 ; 95 \% \mathrm{CI}=2.42-25.72 ; P=0.001$ ).

The most important factor that predicted overall QoL in the MTC + group was the personality characteristic neuroticism. It explained up to $26 \%$ of the variance in $\mathrm{QoL}$ scores (Table 3). Neuroticism is the personality factor that predicted overall QoL in the MTC + group and thus was included in the logistic regression analyses. For the MTC + group, a high score on neuroticism had a profound and negative influence on overall QoL. The chance that women in the MTC + group with a high score on neuroticism experienced a low QoL 6 months postsurgery was 10.3 $(\mathrm{OR}=10.26 ; 95 \% \quad \mathrm{CI}=2.05-51.43 ; P=0.0001)$ and
1 year postsurgery $4.4(\mathrm{OR}=4.38 ; 95 \% \mathrm{CI}=1.02-18.90$; $P=0.048)$.

The percentage of patients with a high score on trait anxiety did not differ between the patient groups. With respect to neuroticism, the percentage of women scoring high was low in the conversion group compared with the BCT and MTC groups, but the scores did not differ significantly (Table 2).

Using general linear models, the impact of trait anxiety and neuroticism on the scores of overall QoL was assessed. The women were divided into four groups based on their surgical treatment and their scores on one of these two personality factors (Fig. 2). Women with high scores on trait anxiety had significantly lower QoL scores at all measurement moments irrespective of surgical treatment $\left(P<0.001, \eta^{2}=0.223\right.$, observed power $\left.=0.99\right)$. However, the differences in QoL scores were most pronounced for the BCT group. Women in this group with a low score on trait anxiety had the highest QoL scores and the BCT women with a high score on trait anxiety had the worst QoL scores $(P<0.002$ for all measurement moments). Women with high scores on neuroticism scored significantly lower on overall QoL, again irrespective of surgical treatment $\left(P=0.024 ; \eta^{2}=0.09\right.$, observed power $=0.74)$.

\section{Discussion}

The aim of the present study was to establish the influence of surgical treatment and personality on overall QoL. A difference in overall QoL in favor of the BCT group was expected. Previous studies have shown that body image is better after BCT and that physical complaints tend to be
Table 3 Regression analyses for overall QoL as dependent variable for both treatment groups

\begin{tabular}{llllrr}
\hline Measurement moment & Treatment & Independent factor & $R^{2}$ & \multicolumn{1}{r}{$\beta$} & $P$ value \\
\hline T2 & BCT & Trait anxiety & 0.29 & -0.53 & $<0.001$ \\
& MTC + & Neuroticism & 0.19 & -0.43 & $<0.001$ \\
T3 & BCT & Trait anxiety & 0.37 & -0.61 & $<0.001$ \\
& & Conscientiousness & 0.09 & 0.30 & 0.004 \\
& \multirow{3}{*}{ MTC +} & Agreeableness & 0.04 & 0.23 & 0.037 \\
& & Neuroticism & 0.21 & -0.45 & $<0.001$ \\
& \multirow{3}{*}{ BCT } & Radiotherapy & 0.08 & -0.28 & 0.009 \\
& & Trait anxiety & 0.43 & -0.66 & $<0.001$ \\
& & Education & 0.11 & 0.33 & 0.005 \\
& \multirow{3}{*}{ MTC +} & Agreeableness & 0.06 & 0.24 & 0.015 \\
& & Neuroticism & 0.20 & -0.44 & $<0.001$ \\
& BCT & Radiotherapy & 0.10 & -0.31 & 0.004 \\
& & Neuroticism & 0.34 & -0.58 & $<0.001$ \\
& MTC + & Education & 0.09 & 0.30 & 0.011 \\
& & Neuroticism & 0.26 & -0.51 & $<0.001$ \\
\hline
\end{tabular}

$R^{2}$ is the percentage of variance in the scores of the dependent variable explained by the independent variable $(1.00=100 \%)$. A negative beta means that a higher score of the independent variable will result in a lower score of the dependent variable 

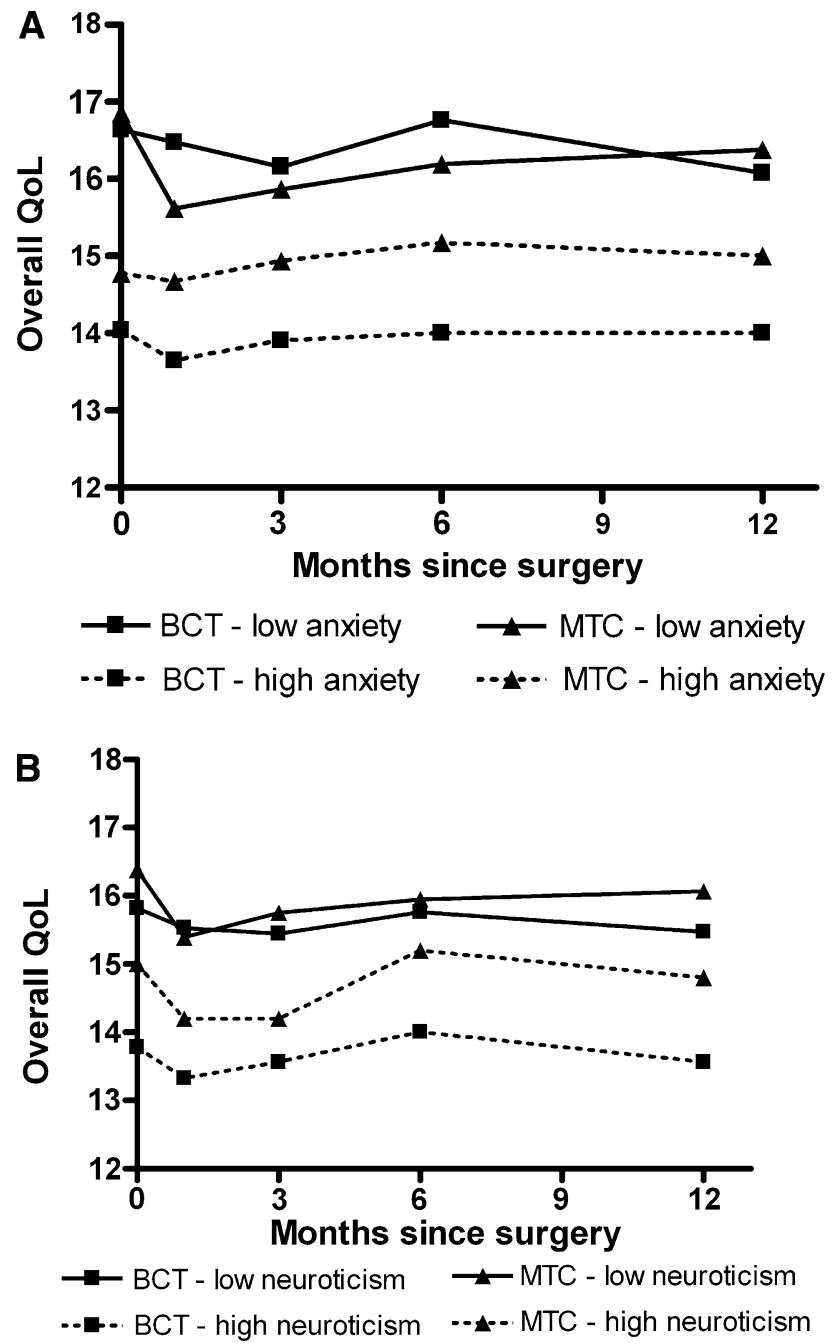

Fig. 2 Scores on overall quality of life and general health for a trait anxiety and $\mathbf{b}$ neuroticism. Scores are represented as means. Patients were divided into four groups based on their surgical treatment (BCT vs. MTC) and their scores on the given personality trait (high score vs. not-high score) In (a), the standard error of the means (SEM) did not exceed 0.6. In (b), the SEM was on measurement moment 4 between 1.0 and 1.3; for the other moments it did not go higher than 0.3

less severe following BCT [19]. However, in the present study no significant differences were observed between the two treatment groups for either the scores on overall $\mathrm{QoL}$ or the QoL domain scores. The contrast of these results with expectations may be explained by the questionnaires used to measure QoL. When analyzing previous studies concerning QoL in breast cancer patients, the vast majority did not use QoL questionnaires but measured health status (HS) [20]. QoL and HS are often considered interchangeable, but the concepts differ considerably. HS reflects solely the functioning in the physical, psychological, and social domains and does not focus on the subjective satisfaction of the individual [21], whereas QoL revolves entirely around the contentment with functioning. Applying a HS questionnaire and a QoL questionnaire to the same study population will lead to different outcome scores [22].

With regard to the influence of personality on overall QoL scores, a profound influence of anxiety was expected due to fear of recurrence of cancer. Nowadays, there is general agreement about the view that personality, at least for descriptions at a rather global level, can be described adequately in terms of the Big Five dimensions: neuroticism, extraversion, openness to experience, agreeableness, and conscientiousness [23].

Neuroticism is an important and extensive domain. It weighs emotional instability against emotional stability, i.e., the tendency to experience no distressing emotions such as fear, guilt, and frustration. Extraversion concerns the degree in which energy, orientation, and attention are focused on the outside world in contrast to the inner world. Openness refers to an open attitude towards other people, beliefs, and experiences. Agreeableness represents one's orientation towards experiences, goals, and interests of other people. Conscientiousness refers directly to the conscience as a guiding and analyzing instrument for one's own behavior [13]. Anxiety can be considered a part of the neuroticism domain, but it can also be defined individually. Trait anxiety refers to the tendency to respond to situations perceived as threatening with a rise in anxiety intensity [14].

As expected, trait anxiety was indeed a strong predictor of overall QoL, but only in the BCT group. When women were divided into four groups based on their scores on anxiety and surgical treatment, trait anxiety turned out to have more impact on QoL than surgical treatment. These findings imply that the beneficial effects of BCT on body image and presumably on overall $\mathrm{QoL}$ are negated in women who experience anxiety.

For the MTC + group, the personality trait neuroticism had significant influence on the overall QoL and, again, this influence exceeded the influence of surgical treatment. However, the effect of neuroticism on overall quality of life was not as strong as the effect of trait anxiety.

It may be that the 28 women who chose a BCT but ended up with a mastectomy influenced the aforementioned results. However, we would expect these women to have a negative influence on the outcomes of the MTC + group. Instead, lower scores were found in the BCT group. In addition, no significant differences were found between the three patient groups (BCT, conversion, and MTC) at baseline. Therefore, we feel that this potential bias did not influence the results presented.

Previous studies have shown that anxiety is a determining factor in QoL. Fear of the future, fear of dying, and fear of recurrence of cancer all have a profound influence 
on QoL [24]. Several studies have looked into personality and psychological health in women with breast cancer and confirmed a negative influence of depression and anxiety [24-26] and neuroticism [27, 28] on QoL and psychosocial adjustment to the diagnosis breast cancer. However, none of these studies included surgical treatment as a variable of influence.

Studies performed by Ormel et al. [29, 30] showed that personality is more powerful for predicting psychological distress than external events such as diseases or inadvertent life events. Thus, the strong influence of personality on subjective well-being in general and on coping mechanisms in the case of adverse life events has now been found for breast cancer patients as well.

In conclusion, QoL in breast cancer patients is not significantly influenced by surgical treatment but mainly by personality, especially the factors of trait anxiety, neuroticism, and extraversion. The diminished QoL in BCT patients who score high on trait anxiety is especially important since these findings bring up questions about treatment choice. Women in the BCT group turned out to have smaller tumors, less axillary metastases, and, thus, less adjuvant treatment. The fact that they had a less mutilating surgical procedure compared with MTC, would not experience the adverse effects of adjuvant treatment, and, statistically speaking, would have the best prognosis concerning survival, should result in a better QoL. However, this better QoL could not be confirmed, and, even worse, women with certain personality traits would have been better off having MTC. So far, clinics have been judged by the percentage of breast-conserving surgeries performed for early-stage breast cancer. However, in the decision process the personality of women diagnosed with breast cancer has never been taken into consideration. Based on our results, we feel that personality factors should be incorporated into the advice given to women when they choose between MTC and BCT and that women scoring high on trait anxiety should be made aware of the possible negative influence of their personality on their experienced QoL when they choose BCT. When these women choose BCT after being well informed, it is important to offer them psychosocial support and counseling to help them live and cope with their fears. Future studies that look at personality factors and evaluate psychosocial interventions are needed to examine the generalizability of our results.

Acknowledgments The authors like to acknowledge Dr FWC van der Ent and Dr MF Ernst for their help in accrual of patients.

Open Access This article is distributed under the terms of the Creative Commons Attribution Noncommercial License which permits any noncommercial use, distribution, and reproduction in any medium, provided the original author(s) and source are credited.

\section{References}

1. Poortmans PMP (2005) Quality assurance in clinical trials in breast cancer. Drukkerij Bulckens NV, Herentals

2. Veronesi U, Cascinelli N, Mariani L et al (2002) Twenty-year follow-up of a randomized study comparing breast-conserving surgery with radical mastectomy for early breast cancer. N Engl J Med 347(16):1227-1232

3. Keynes G (1929) The treatment of primary carcinoma of the breast with radium. Acta Radiol 10:393-402

4. Mustakallio S (1954) Treatment of breast cancer by tumor extirpation and roentgen therapy instead of radical operation. J Faculty Radiol 6:23-26

5. WHOQOL group (1994) Development of the WHOQOL: rationale and current status. Int J Ment Health 23:24-56

6. Diener E, Oishi S, Lucas RE (2003) Personality, culture, and subjective well-being: emotional and cognitive evaluations of life. Ann Rev Psychol 54:403-425

7. van der Steeg AFW, De Vries J, van der Ent FWC, Roukema JA (2007) Personality predicts quality of life six months after the diagnosis and treatment of breast disease. Ann Surg Oncol 14(2):678-685

8. Irwig L, Bennetts A (1997) Quality of life after breast conservation or mastectomy: a systematic review. Aust N Z J Surg 67:750-754

9. WHOQOL group (1995) The World Health Organization Quality of Life assessment instrument. WHO, Geneva

10. De Vries J, Van Heck GL (1995) De Nederlandse versie van de WHOQOL-100 [The Dutch version of the WHOQOL-100]. Tilburg University, Tilburg

11. Den Oudsten BL, Van Heck GL, Van der Steeg AFW et al (2009) The WHOQOL-100 has good psychometric properties in breast cancer patients. J Clin Epidemiol 62(2):195-205

12. Costa PT Jr, McCrae RR (1989) NEO-PI/FFI manual supplement. Psychological Assessment Resources, Odessa, FL

13. Hoekstra HA, Ormel J, de Fruyt F (1996) Manual for the NEO Personality Inventories NEO-PI-R and NEO-FFI. Swets \& Zeitlinger B.V., Lisse, The Netherlands

14. Spielberger CD, Gorsuch RL, Lushene RE (1970) The State-Trait Anxiety Inventory manual. Consulting Psychologists Press, Palo Alto, CA

15. van der Ploeg HM, Defares PB, Spielberger CD (1980) A Dutchlanguage adaptation of the Spielberger State-Trait Anxiety Inventory. Swets \& Zeitlinger B.V., Lisse, The Netherlands

16. Costa PT Jr, McCrae RR (1992) NEO-PI-R: professional manual. Psychological Assessment Resources, Odessa, FL

17. Spielberger CD, Gorsuch RL, Lushene RE (1968) State-Trait Anxiety Inventory, Preliminary test manual for form X. Florida State University, Tallahassee, FL

18. Cohen JW (1988) Statistical power analysis for the behavioral sciences, 2nd edn. Lawrence Erlbaum Associates, Hillsdale, NJ

19. Ganz PA, Kwan L, Stanton AL et al (2004) Quality of life at the end of primary treatment of breast cancer: first results from the moving beyond cancer randomized trial. J Natl Cancer Inst 96(5):376-387

20. van der Steeg AFW, De Vries J, Roukema JA (2004) Quality of life and health status in breast carcinoma. Eur J Surg Oncol 30(10): 1051-1057

21. De Vries J (2001) Quality of life assessment. In: Vingerhoets AJJM (ed) Assessment in behavioral medicine. Brunner-Routledge, Hove, pp 353-370

22. Breek JC, de Vries J, van Heck GL et al (2005) Assessment of disease impact in patients with intermittent claudication: discrepancy between health status and quality of life. J Vasc Surg 41(3):443-450 
23. McCrae RR, John OP (1992) An introduction to the five-factormodel and its applications. J Pers 60(2):175-215

24. Fredette SL (1995) Breast cancer survivors: concerns and coping. Cancer Nurs 18:35-46

25. Bleiker EM, Pouwer F, Van der Ploeg HM et al (2000) Psychological distress two years after diagnosis of breast cancer: frequency and prediction. Patient Educ Couns 40:209-217

26. Longman AJ, Braden CJ, Mishel MH (1999) Side-effects burden, psychological adjustment, and life quality in women with breast cancer: pattern of association over time. Oncol Nurs Forum 26:909-915

27. Golden-Kreutz DM, Andersen BL (2004) Depressive symptoms after breast cancer surgery: relationships with global, cancerrelated, and life event stress. Psychooncology 13:211-220
28. Van der Zee K, Buunk B, Sanderman R (1998) Neuroticism and reactions to social comparison information among cancer patients. J Pers 66:175-194

29. Ormel J, Schaufeli WB (1991) Stability and change in psychological distress and their relationship with self-esteem and locus of control: a dynamic equilibrium model. J Pers Soc Psychol 60:288-299

30. Ormel J, Wohlfart T (1991) How neuroticism, long-term difficulties, and life situation change influence psychological distress: a longitudinal model. J Pers Soc Psychol 60:744-755 